\title{
Complications of the naevoid basal cell carcinoma syndrome: results of a population based study
}

\author{
D G R Evans, E J Ladusans, S Rimmer, L D Burnell, N Thakker, P A Farndon
}

\begin{abstract}
There are many potential complications which have been reported in association with the naevoid basal cell carcinoma syndrome. We have been able to show the relative frequencies of these problems in a population based study of 84 cases in the north west of England. The major complications of basal cell carcinomas and jaw cysts occur in over $90 \%$ of patients by 40 years of age, but may both occur before 10 years of age. Less well described complications are ovarian calcification or fibroma (24\%), medulloblastoma $(5 \%)$, cardiac fibroma (3\%), cleft palate (5\%), and ophthalmic abnormalities such as squint or cataract $(26 \%)$. This study more clearly defines the possible complications of the syndrome and gives clearer guidelines for counselling and screening affected and at risk persons.
\end{abstract}

( $(\mathcal{F}$ Med Genet 1993;30:460-4)

Department of Medical Genetics, St Mary's Hospital, Hathersage Road Manchester M13 0JH,

UK.

D G R Evans

L D Burnell

N Thakker

Department of Paediatric Cardiology, St Mary's Hospital, Manchester M13 0JH, UK.

E J Ladusans

Department of Radiology, St Mary's Hospital, Manchester M13 0JH, UK. M13 0JH,
S Rimmer

Department of Medical Genetics, Birmingham Maternity Hospital, Edgbaston, Birmingham, UK.

P A Farndon

CRC Department of Cancer Genetics,

Christie Hospital, Manchester M20 9BX, UK.

D G R Evans

L D Burnell

Correspondence to Dr Evans. Received 24 June 1992. Revised version accepted 10 September 1992.
The naevoid basal cell carcinoma syndrome (NBCCS) was reported as early as $1894,{ }^{12}$ but was not appreciated as a distinct entity until the latter part of this century. ${ }^{34}$ The syndrome was delineated by Gorlin and Goltz in $1960^{4}$ and often bears the former's name. A recent exhaustive review by the same author ${ }^{5}$ covers all the known features of the condition. NBCCS is an autosomal dominant condition characterised by the development of basal cell carcinomas in the third decade onwards and jaw cysts in the second and third decades. Skeletal anomalies are a consistent feature and the more commonly found of these are bifid ribs, vertebral anomalies, Sprengel shoulder, and short fourth metacarpal. The gene has recently been mapped to chromosome $9^{6}$ with no apparent heterogeneity. The complications of NBCCS are well known, but the relative frequencies of the rarer problems have only been informed guesses. The lack of a large population based study to assess these frequencies and the requirement for screening led us to undertake a study in the north west of England.

\section{Patients and methods}

The study was started in 1982 (by PAF) when all dermatology, oral, and plastic surgery departments in the North West Regional Health Authority area (population 4001000 ) were contacted to obtain information about patients with NBCCS. This study ascertained 27 fami- lies and all close relatives of the index patient were contacted and examined where possible. This arm of the study ended in autumn 1983 . The diagnostic criteria used in this study are shown in table 1. Subjects were asked about relevant past medical and dental history such as removal of a jaw cyst, 'abscess', or skin lumps. Any relevant family history such as childhood brain tumours was also sought. Full clinical examination included head circumference, interpupillary distance, and careful examination of the skin for basal cell naevi or carcinomata as well as cysts and pits in the palms and soles. $X$ rays were taken of the chest, AP and lateral skull, jaw (orthopantogram), spine, and hands. In March 1990 the families were recalled with the setting up of an NBCCS family register. Further attempts were made to ascertain new affected family members and families, by recontacting referring departments and including paediatric departments. In addition to the previous protocol all female family members were offered an ovarian ultrasound scan and pelvic $x$ ray and all children underwent an echocardiogram.

\section{Results}

A total of 29 families with 72 living members has been ascertained in the study period 1982 to February 1992. This represents a prevalence of 1 in 55600 (on prevalence day 1 January 1991). Information on a further 12 family members who have died was also available.

Table 1 Diagnostic criteria for naevoid basal cell carcinoma syndrome.

A diagnosis can be made when 2 major or 1 major and 2 minor criteria are fulfilled.

Major criteria

(1) Multiple $(>2)$ basal cell carcinomas or one under 30 years, or $>10$ basal cell naevi.

(2) Any odontogenic keratocyst (proven on histology), or polyostotic bone cyst.

(3) Palmar or plantar pits (3 or more)

4) Ectopic calcification: lamellar or early ( $<20$ years) falx calcification.

(5) Family history of NBCCS

Minor criteria

(1) Congenital skeletal anomaly: bifid, fused, splayed, or missing rib, or bifid, wedged, or fused vertebra.

(2) OFC $>97$ th centile, with frontal bossing.

(3) Cardiac or ovarian fibroma.

(4) Medulloblastoma

(5) Lymphomesenteric cysts.

(6) Congenital malformation: cleft lip and/or palate, polydactyly, eye anomaly (cataract, coloboma, microphthalmia). 
SKIN COMPLICATIONS

Basal cell carcinomas (BCC) had been removed from $33 / 70(47 \%)$ cases but naevi were present in all but two cases over 20 years of age. Only $12 / 45(27 \%)$ cases over 20 years and $2 / 21(9.5 \%)$ cases over 40 years of age had not developed a BCC. Several younger patients were noted to have multiple naevi all over the trunk and limbs which were present only shortly after puberty. Three cases have had BCCs removed before 20 years of age; one case who required craniospinal radiotherapy for a medulloblastoma at the age of 29 months started to develop BCCs only 18 months later. The latter case was not included in the 70 cases as he was not examined by the authors and has since died. This case and $4 / 70$ of the examined cases have had extensive surgery for BCCs on the face, resulting in major rhinoplasty, enucleation of eyes, pinnectomies, and skin grafts. Three out of five of these severe cases had received radiation therapy, either for the BCCs or for a medulloblastoma.

Pits were present in the palms and soles in $50 / 70$ cases $(71 \%$ ) but no case developed a $\mathrm{BCC}$ at these sites.

\section{JAW CYSTS}

Odontogenic keratocysts had been detected and removed in 46/70 (66\%) cases. Only 8/45 $(18 \%)$ cases over 20 years and $2 / 21(9.5 \%)$ cases over 40 years of age have not developed this complication. Cysts were detected in two cases under 10 years of age (at 9 years), but were also present in edentulous jaws. The cysts were, however, much less common over 30 years of age. No cysts were known to have undergone malignant transformation.

\section{CENTRAL NERVOUS SYSTEM}

Medulloblastoma occurred in 2/84 cases and was suspected in a third case who died with a posterior fossa tumour, but no histological confirmation. These tumours occurred at 15 , 29 , and 48 months of age. The two confirmed cases survived their tumour, but were mentally retarded partly as a result of their therapy. The only other CNS tumours to occur were a glioblastoma multiforme in the father of one of the medulloblastoma cases and a meningioma which was removed from a further case in adulthood. Apart from the treated cases of medulloblastoma there were no cases of moderate or severe mental retardation. Also excluding the cases with brain tumour $5 / 84$ cases required prolonged anticonvulsant therapy for grand mal seizures.

\section{GENITOURINARY SYSTEM}

Twenty-five asymptomatic women with NBCCS underwent abdominal ultrasound of their ovaries and had pelvic $x$ rays performed. Four of these cases were noted to have calcification within the ovaries and one of these had a clearly defined large ovarian fibroma (figure). This case and a further case in whom no abnormality was seen on radiological imaging

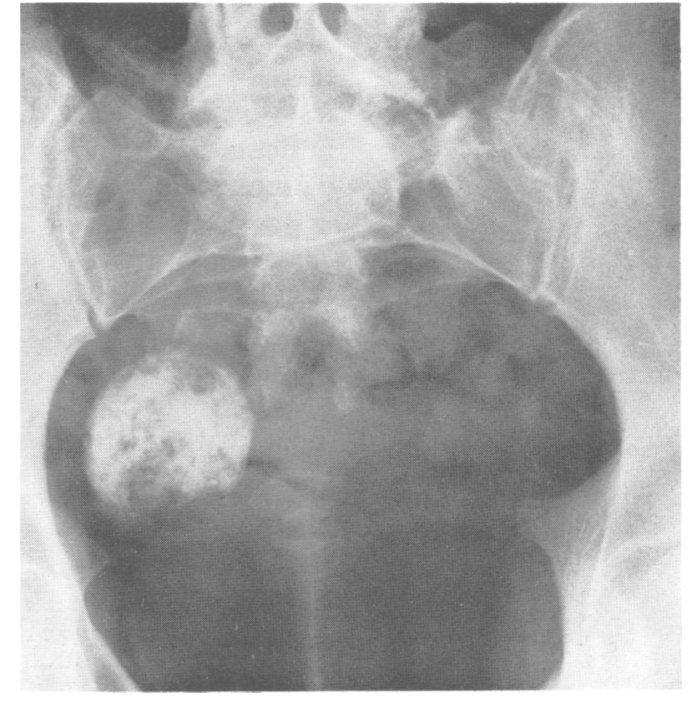

Abdominal $x$ ray in a woman with NBCCS showing a large calcified ovarian fibroma confirmed at laparoscopy.

were seen to have fibromas present at laparoscopy and opportunistic examination during a caesarian section respectively. As well as the cases scanned a further patient had undergone bilateral oophorectomy for massive bilateral calcified ovarian fibromas. As no woman has so far refused a scan there was evidence for the presence of an ovarian fibroma in $6 / 25$ cases $(24 \%)$. No case of malignant transformation was found and no other genitourinary malignancy was discovered.

\section{CARDIOVASCULAR SYSTEM}

Fourteen asymptomatic children known to have NBCCS and three equivocal cases have undergone a doppler echocardiogram examination. A further case who has given birth to a boy thought to have NBCCS underwent a full fetal anomaly scan during pregnancy. No cases of cardiac fibroma have been found in this series. However, one case known to have NBCCS died at 3 months of age from multiple cardiac fibromas. A further case has been followed up for over 20 years with a single $2 \mathrm{~cm}$ cardiac fibroma in the interventricular septum. This has remained unchanged throughout this period.

\section{RESPIRATORY SYSTEM}

One case presented in his twenties with dyspnoea owing to a large bronchogenic cyst. A premature neonate died from hyaline membrane disease but the chest size was significantly reduced by the presence of multiple rib anomalies.

\section{EYE COMPLICATIONS}

Eighteen out of $70(26 \%)$ cases personally examined had problems with their eyes. Ten cases had a convergent strabismus and three cases from the same family had rotatory nystagmus. Cataracts were present in four cases and microphthalmia in a single case. 
NEOPLASIA IN OTHER ORGANS

The only other neoplasm which has been linked with NBCCS that occurred in this series was Hodgkin's disease in one case. Two patients died from lung cancer and a further patient from renal cell carcinoma.

\section{CLEFT LIP AND PALATE}

A cleft of the lip and palate was present in 4/70 cases $(5 \cdot 7 \%)$.

A summary of the frequencies of the various complications from this study is shown in table 2 .

\section{Discussion}

This is the first large population based study of NBCCS and as such should provide a good indication of the relative frequencies of the complications of the condition. We have been able to ascertain patients from their dermatological, dental, neoplastic, and chance skeletal findings. It is thus hoped that as little bias as possible has entered the study, as all modes of presentation are covered. Although we cannot be sure of $100 \%$ ascertainment as mild cases may not present with any complication, the results should give a good guide for anyone dealing with a 'known' case. Unfortunately as several families contained more than one index case, exclusion of these would have reduced the sample size to only around 30 living cases. Complications were, however, relatively similar within families and we do not feel that failure to exclude index cases will have increased the apparent complication rate. For example, there were index cases elsewhere in the family for both cardiac tumour patients and $2 / 3$ medulloblastoma patients.

BCCs are one of the major criteria of NBCCS and as such occur in nearly $75 \%$ of patients over 20 years and more than $90 \%$ over 40 years of age in our study. This correlates well with estimates that only $10 \%$ of cases do not manifest the skin lesions after 30 years of age. ${ }^{5}$ The BCCs are unlikely to occur under the age of puberty unless the patient has been exposed to radiotherapy, but cases as early as 2 years have been described. ${ }^{7}$ The real acceleration in growth appears to occur in the third and fourth decade. Radiotherapy should be avoided if at all possible $e^{58}$ although some patients appear to be unaffected by it. Tumours on the scalp can be particularly aggressive $^{910}$ and need early intervention. Care must

Table 2 Complication rates in 84 patients with NBCCS

\begin{tabular}{|c|c|}
\hline 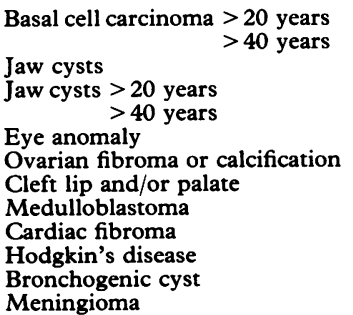 & $\begin{array}{l}73 \% \\
90 \cdot 5 \% \\
66 \% \\
82 \% \\
90 \cdot 5 \% \\
26 \% \\
24 \% \\
5 \cdot 7 \% \\
5 \% \\
2.5 \% \\
1 \% \\
1 \% \\
1 \%\end{array}$ \\
\hline
\end{tabular}

also be taken over lesions around the eyelids ${ }^{5}$ as loss of the eye may occur as in one of our cases. Although BCCs occur particularly in the facial region they can occur on nonexposed areas and have even been found in the labia..$^{5}$ Although NBCCS patients appear to be particularly sensitive to $x$ irradiation and ultraviolet light, attempts to show this at the cellular level have been largely unsuccessful. ${ }^{1011}$

Odontogenic keratocysts had occurred in over $80 \%$ of patients over 20 years old in our study. This is similar to previous estimates. ${ }^{5}$ They occurred in two patients under 10 years in our study and are known to develop in the first decade. ${ }^{12}$ They are rarely symptomatic even though they are large and unless detected early loss of teeth and more severe complications such as pathological fracture ${ }^{13}$ may occur. Jaw malignancy has been described in rare cases, ${ }^{14}$ but was not found in our study. The cysts have a high recurrence rate and new cysts are likely to occur, with the mandible the commonest site. ${ }^{15}$

Medulloblastoma is now a well recognised complication of NBCCS and occurs at an average age of 2 years as opposed to $7 \cdot 26$ years in the general population. ${ }^{8}$ The early age at onset in NBCCS may reflect the fact that the NBCCS gene is acting as a tumour suppressor as has been shown for BCCs. ${ }^{16}$ This study has been able to provide the first good estimate of its frequency $(5 \%)$. A family history and careful examination of medulloblastoma cases, especially if of early onset, is recommended so that the inevitable crop of BCCs after radiotherapy can be anticipated. There is no clear evidence for an increased incidence of other brain tumours although meningioma has also been reported before. ${ }^{17}$

Ovarian fibromas were first documented in NBCCS in $1964^{14}$ and have been estimated to occur in up to $75 \%$ of all NBCCS patients attending a gynaecology department. ${ }^{5}$ No accurate estimate of their true prevalence in the NBCCS population has been possible. We have been able to assess this as occurring in between 12 and $24 \%$ of patients. The tumours are usually totally asymptomatic as shown by the absence of problems in 5/6 of our cases. They can, however, twist on their pedicle and cause infarction of the ovary, which has been documented as occurring as early as $3 \frac{1}{2}$ years of age. ${ }^{18}$ The tumours are not thought to interfere with fertility and rarely undergo malignant change ${ }^{19}$ and no evidence of this was found in our study. Although the tumours are asymptomatic knowledge of them may prevent loss of an ovary by delay in the patient presenting or in diagnosis of a torted ovary. It is certainly sensible to check the ovaries in cases who have undergone craniospinal irradiation as this is a situation in which malignant transformation may occur. If detecting the tumour on scan is only going to cause the patient to be submitted to an unnecessary laparotomy then screening is not recommended.

Cardiac fibromas are very rare, constituting only $5 \%$ of cardiac neoplasms. ${ }^{5}$ Our second case has been reported before, ${ }^{20}$ but with one new report here and another recent report ${ }^{21}$ the 
presence of these tumours in at least 14 cases with NBCCS is now certainly significant. They appear to present in childhood with $85 \%$ in children under 10 years of age, with the interventricular septum the most common site. $^{22}$ Little is known about the natural history, although asymptomatic tumours have been described in association with NBCCS. ${ }^{23}$ We have looked specifically for these tumours in children to see if they follow a similar course to the cardiac tumours in tuberous sclerosis by regressing with age. The absence of the tumours in our asymptomatic series is encouraging, but they can be severe enough to kill the patient as in our 3 month old. The presence of a tumour which has remained unchanged since childhood in our other case suggests that these tumours develop very early, probably in fetal life, but do not progress after puberty. Patients may remain well under observation ${ }^{20}$ or after removal of the tumour ${ }^{24}$ or may die from unresectable tumours. ${ }^{25}$

Abnormalities of the eye have not previously been thought to be a major part of NBCCS; Gorlin ${ }^{5}$ estimated that the frequency of cataract, glaucoma, and coloboma combined was less than $14 \%$. We have clearly shown a significant level of anomalies in our population based series $(26 \%)$. We have therefore included these as minor criteria in our diagnostic criteria (table 1). Cleft palate was also found in excess frequency as noted before. ${ }^{5}$

Other complications of NBCCS are rare, although we have purposely not alluded to the common skeletal anomalies as these are largely hidden and usually completely asymptomatic. The case with the bronchogenic cyst has been reported before ${ }^{26}$ and Hodgkin's disease has also been previously reported in association with NBCCS. ${ }^{27}$

CARE OF THE PATIENT WITH NBCCS

It is recommended that families with NBCCS are managed by a register system so that regular screening can be ensured and the patient kept up to date with recent advances. The linkage of the NBCCS gene to chromosome $9^{6}$ opens the way for diagnosis in equivocal cases and for prenatal testing if this is desired. Accordingly an interested genetics service would be the best place to run the register and coordinate screening. Screening can begin during pregnancy with the offer of a fetal anomaly scan to a person with NBCCS or who has an affected partner. This could detect cardiac tumours and developmental anomalies such as cleft palate. Scans may also detect the enlarged head which may necessitate a caesarian section or induction of early labour. At birth, or soon after, a detailed examination of a child at $50 \%$ risk would indicate the presence of a large head, cleft palate, or eye anomaly and $x$ rays bifid ribs or vertebral anomalies. An echocardiogram is best performed early as at least two cases have presented before 3 months of age with fibromas. Six monthly neurological examination is recommended to detect a deficit which may indicate a medulloblastoma. Routine scanning with CT and indeed excessive use of $x$ rays of any kind is not advisable because of the risk of inducing a malignancy. At 3 years the examinations could be reduced to annually until 7 years after which a medulloblastoma is very unlikely. Although these examinations are of low sensitivity and specificity a parent will at least have contact with a specialist department, should suspicious symptoms develop. At 8 years annual dental screening should be started and this will usually entail an orthopantogram of the jaw. Annual examination of the skin from puberty is also recommended although this and the dental checks may need to be increased if active lesions are present. Patients may well be under dental and dermatology departments in which case the register would merely act as a reminder and furnisher of information. Although this is a fairly aggressive screening protocol, most affected subjects would only need to be seen once or twice per year. With the help of DNA tests most even equivocal cases could be assigned as affected or unaffected before puberty and the risk of childhood complications may justify predictive testing of minors. This means that unaffected subjects can be removed from the screening protocol as early as the neonatal period. We do, however, feel that regular contact and screening will reduce the effect of the often devastating complications of this disorder and prevent potentially harmful treatments such as radiotherapy for BCCs.

1 Jarisch W. Zur Lehre von den Hautgeschwulsten. Arch Dermatol Syphilol (Berl) 1894;28:162-222.

2 White JC. Multiple benign cystic epitheliomas. 7 Cutan Genitourin Dis 1894;12:477-84.

3 Binkley GW, Johnson HH. Epithelioma adenoides cysticum: basal cell nevi, agenesis of corpus callosum and dental cysts. Arch Dermatol Syphilol (Berl) 1951;63:7384.

4 Gorlin RJ, Goltz RW. Multiple nevoid basal cell epithelioma, jaw cysts and bifid rib syndrome. $N$ Engl $\mathfrak{f} \mathrm{Med}$ 1960;262:908-12.

5 Gorlin RJ. Nevoid basal cell carcinoma syndrome. Medicine (Baltimore) 1987;66:99-109.

6 Farndon PA, Del Mastro RG, Evans DGR, Kilpatrick $M W$. Localisation of the gene for Gorlin syndrome (naevoid basal cell carcinoma syndrome) on the long arm of chromosome 9. Lancet 1992;339:581-2.

7 Gilhuus-Moe O, Haugen LK, Dee PM. The syndrome of multiple cysts of the jaws, basal cell carcinomata and skeletal anomalies. Br f Oral Surg 1968;5:211-22.

8 Evans DGR, Farndon PA, Burnell LD, Gattameneni R, Birch J. The incidence of Gorlin syndrome in 173 consecutive cases of medulloblastoma. Br $₹$ Cancer 1991; 64:959-61.

9 Evans DGR, Birch J, Orton C. Brain tumours and the occurrence of severe invasive basal cell carcinomas in first degree relatives with Gorlin syndrome. $\mathrm{Br} \mathcal{F}$ Neurosurg 1991;5:643-6.

10 Clendenning WE, Block JB, Radde IC. Basal cell nevus syndrome. Arch Dermatol 1964;90:38-53.

1 Nagasawa H, Little FF, Burke MJ, et al. Study of basal cell nevus fibroblasts after treatment with DNA damaging agents. Basic Life Sci 1984;29B:775-85.

12 McClatchet K, Batsakis JG, Hybels R, Van Wieren CR. Odontogenic keratocysts and nevoid basal cell carcinoma syndrome. Arch Otolaryngol 1975;101:613-16.

13 Leppard BJ. Skin cysts in the basal cell naevus syndrome. Clin Exp Dermatol 1983;8:603-12.

14 Clendenning WE, Block JB, Radde IC. Basal cell nevus syndrome. Arch Dermatol 1964;90:38-53.

15 Brannon RB. The odontogenic keratocyst: a clinicopathological study of 312 cases. Part II. Histologic features. Oral gical study of 312 cases.

16 Gailani M, Leffell DJ, Bale AE. Evidence for a tumour suppressor gene on chromosome 9 in basal cell carcinomas of the skin. Am f Hum Genet (Suppl) 1991;49:454

17 Dawber RPB, Ryan TJ. Basal cell nevus syndrome and malignant meningioma. $\mathrm{Br} f$ Dermatol 1980;103 (suppl 18):42

18 Johnson AD, Hebert AA, Esterly NB. Nevoid basal cell carcinoma syndrome: bilateral ovarian fibromas in a $3 \frac{1}{2}$ year old girl. $\mathcal{f}$ Ann Acad Dermatol 1986;14:371-4.

19 Strong LC. Genetic and environmental interactions. Cancer 1977;40:1861-6.

20 Harris SA, Large DM. Gorlin's syndrome with a cardiac 
lesion and jaw cysts with some unusual histological features: a case report and review of the literature. Int $f$ Oral Surg 1984;64:13-59.

21 Herman TE, Siegel MJ, McAlister WH. Cardiac tumour in Gorlin syndrome. Pediatr Radiol 1991;21:234-5.

22 Williams DB, Danielson GK, McGoon DC, Feldt RH, Edwards WD. Cardiac fibroma: long term survival after excision. $\mathcal{F}$ Thorac Cardiovasc Surg 1982;84:230-6.

23 Bunting PD, Remensnyder JP. Basal cell nevus syndrome. Plast Reconstr Surg 1977;60:895-901.

24 Littler BO. Gorlin's syndrome and the heart. Br $\mathrm{f} \mathrm{Oral}$ Surg 1979;17:135-46.
25 Jones KL, Wolf PL, Jensen P, Dittrich H, Benirschke K, Bloor C. The Gorlin syndrome: a genetically determined disorder associated with cardiac tumour. Am Heart $\mathcal{f}$ 1986;111:1013-15.

26 Totten JR. The multiple nevoid basal cell carcinoma syndrome: report of its occurrence in four generations of a family. Cancer 1980;46:1456-62.

27 Potaznik D, Steinherz P. Multiple nevoid basal cell carcinoma syndrome and Hodgkin's disease. Cancer 1984;53:2713-15. 